Europhys. Lett., 57 (5), pp. 683-689 (2002)

\title{
Excitation energies from time-dependent density-functional formalism for small systems
}

\author{
F. Aryasetiawan ${ }^{1}$, O. Gunnarsson ${ }^{2}$ and A. Rubio ${ }^{2}$ \\ 1 Joint Research Center for Atom Technology, Angstrom Technology Partnership \\ 1-1-4 Higashi, Tsukuba, Ibaraki 305-0046, Japan \\ and Research Institute for Computational Sciences, Tsukuba Central 2 \\ 1-1-1 Umezono, Ibaraki 305-8568, Japan \\ 2 Max-Planck-Institut für Festkörperforschung - D-70506 Stuttgart, Germany \\ 3 Departamento de Física de Materiales, Facultad de Ciencias Químicas, Universidad \\ del Pais Vasco, Donostia International Physics Center (DIPC) and Centro Mixto \\ CSIC-UPV/EHU - 20018 San Sebastián/Donostia, Basque Country, Spain
}

(received 4 May 2001; accepted in final form 17 December 2001)

PACS. 31.15.Ew - Density-functional theory.

PACS. 31.25.Jf - Electron correlation calculations for atoms and ions: excited states.

PACS. 31.25. $\mathrm{Nj}$ - Electron correlation calculations for diatomic molecules.

\begin{abstract}
As a test of the time-dependent local-density approximation (TDLDA), we study the ${ }^{1} \Sigma_{g}^{+} \rightarrow{ }^{1} \Sigma_{u}^{+}$excitation of $\mathrm{H}_{2}$ as a function of the nuclear distance $d$. We find rather accurate results for intermediate $d$ but not for small and large $d$. At large $d$, TDLDA fails due to the strong non-locality and energy dependence of the exact functional. The spin-dependent formalism gives a qualitative improvement for large $d$. To analyze the results, we compare with the $2 s \rightarrow 2 p$ excitation for $\mathrm{He}, \mathrm{Li}$ and Be for small and intermediate $d$ and with the Hubbard model for large $d$. The fairly accurate results for Li and Be are related to the accuracy of the ground-state formalism for a few electrons.
\end{abstract}

The traditional density functional (DF) formalism [1] is limited to ground-state properties. The time-dependent density-functional formalism [2,3] can, however [3-5], be used to obtain excitation energies. This method has in particular been applied to atoms and molecules [4,6-8]. It has been found that already the time-dependent local density approximation (TDLDA) gives rather accurate results for these systems.

The purpose of this paper is to obtain a better understanding of the TDLDA. We therefore apply the TDLDA to the $\mathrm{H}_{2}$ molecule and study the accuracy as a function of the nuclear separation $d$. Separations away from equilibrium are interesting for the study of dynamical properties of molecules. This system can also give some indications of the accuracy of TDLDA for atoms at surfaces in the so-called surface molecule limit [9]. $\mathrm{H}_{2}$ is a good test case, since there are essentially exact results [10] available, with which we can compare. We find that the accuracy is rather good for intermediate $d$, while it is less good for small $d$. For large $d$ the TDLDA fails qualitatively. The spin-dependent version (TDLSDA) gives a qualitative 


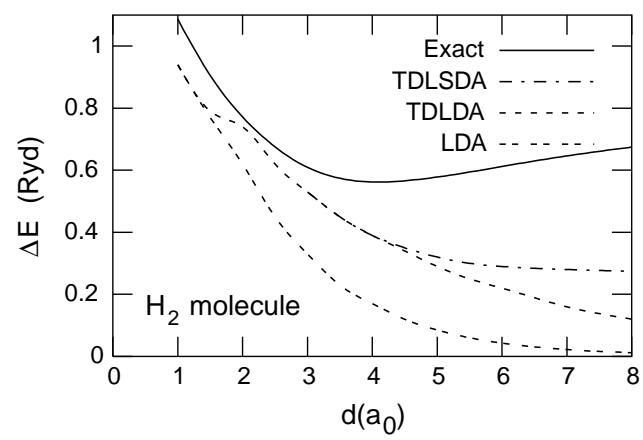

Fig. 1 - The excitation energy $\Delta E$ for the transition from the ${ }^{1} \Sigma_{g}^{+}$state to the ${ }^{1} \Sigma_{u}^{+}$state as a function of the nuclear separation $d$. LDA is the eigenvalue difference in LDA.

improvement in this limit, but is still not very accurate. For large $d$, the results can be understood by comparing an exact and a TDLDA calculation for a two-site Hubbard model. For small $d$ the $\mathrm{H}_{2}$ molecule is closely related to two-electron atoms. We therefore study atoms with one and two valence electrons, and relate the TDLDA to the ground-state local density approximation (LDA) for these systems. The accuracy of the TDLDA for such systems can then be understood in terms of the accuracy of the LDA for the ground-state properties. Similar results for $\mathrm{H}_{2}$ have been obtained earlier by Casida et al. [11] and by Gritsenko et al. [12], but analyzed in different frameworks. These publications also addressed the triplet excitation not treated here. We do not consider gradient corrections, since it has been found that these do not in general improve the response function [13].

To obtain excitation energies, one focuses on the response function $[4,5]$. In matrix form it is given by

$$
\chi=\chi_{0}+\chi_{0}\left(v+f_{\mathrm{xc}}\right) \chi,
$$

where $\chi_{0}$ is the response function of non-interacting electrons moving in the ground-state Kohn-Sham potential, $v$ is the Coulomb interaction and $f_{\mathrm{xc}}(r)=\delta v_{\mathrm{xc}}[\rho] / \delta \rho(r)$ is a functional derivative of the exchange correlation potential. The poles of $\chi$ give excitation energies [4].

We first discuss the treatment of the $\mathrm{H}_{2}$ molecule. The response function $\chi_{0}$ is expressed in terms of the solutions of the Schrödinger-like Kohn-Sham equation in the LDA. Due to the azimuthal symmetry of $\mathrm{H}_{2}$, the ground state only depends on the $z$ - and $\rho$-coordinates, which are discretized on a non-uniform mesh [14]. Expressing the kinetic energy operator as a finite difference, the Kohn-Sham equation is replaced by a matrix problem. The eigenvalues and eigenvectors are found and $\chi_{0}$ is calculated. The full response function $\chi$ is then obtained by inverting a matrix problem corresponding to eq. (1).

The results are shown in fig. 1. For small $d\left(\leq 1.5 a_{0}\right)$ the LDA eigenvalue differences and the TDLDA are similar, both being smaller than the exact result [10]. For these values of $d$, the antibonding state is unbound in the LDA, and therefore extended. TDLDA then gives a small correction to the LDA eigenvalue difference. In the exact calculation, on the other hand, the excited electron is bound (for $d \geq 1$ ). This may be the reason why TDLDA is not so accurate for these separations. This failure of the LDA to give a bound antibonding state is due to the poor cancellation of the electron self-interaction by the exchange-correlation (XC) potential, illustrated by the poor eigenvalue for a free $\mathrm{H}$ atom [15]. For intermediate values of $d$, TDLDA is rather accurate, substantially more accurate than LDA. For large separations the TDLDA fails, giving a much too small excitation energy in agreement with previous results 
obtained by Casida et al. [11] and by Gritsenko et al. [12]. In this limit the LDA eigenvalue difference gives a very poor result, going to zero for $d \rightarrow \infty$. The same should be true for the TDLDA, giving a qualitative failure in this limit.

For $d>3 a_{0}$, the spin-dependent LDA (LSDA) gives a spin-polarized solution, where the two $\mathrm{H}$ atoms have the opposite polarization [16]. Although the spin-polarized solution does not have the correct spin symmetry (not a pure singlet), it describes this strongly correlated situation better in several respects. Figure 1 shows that TDLSDA improves the excitation energy for large $d$, staying finite for $d \rightarrow \infty$. In spite of this qualitative improvement, the TDLSDA result is still much too small.

To discuss large d's, we use a two-site Hubbard model

$$
H=-t \sum_{i, j, \sigma} c_{i \sigma}^{\dagger} c_{j \sigma}+U \sum_{i} n_{i \uparrow} n_{i \downarrow}
$$

where $i$ and $j$ run over the values 1 and $2, t$ is a hopping integral and $U \equiv\langle i i|1 / r| i i\rangle$ is the Coulomb repulsion between two electrons on the same atom. Here

$$
\left\langle i j\left|\frac{1}{r}\right| k l\right\rangle=\int \mathrm{d}^{3} r \mathrm{~d}^{3} r^{\prime} \phi_{i}(r) \phi_{j}(r) \frac{1}{\left|r-r^{\prime}\right|} \phi_{k}\left(r^{\prime}\right) \phi_{l}\left(r^{\prime}\right),
$$

where $\phi_{i}$ is a $1 s$ function on site $i$. The on-site energy is put equal to zero. This model can be obtained by using a basis set consisting of just one $1 s$ state on each atom and neglecting all Coulomb integrals except $U$. For this two-site system, the Coulomb integral $V=\langle 11|1 / r| 22\rangle$ can be easily renormalized into the value of $U, U \rightarrow U-V$ and the neglect of $V$ is therefore not serious. However, there are also other Coulomb integrals, e.g., of the type $J=\langle 12|1 / r| 12\rangle$, which are important for small and intermediate $d$. The Hubbard model is therefore a bad representation of $\mathrm{H}_{2}$ for such values of $d$. We introduce a LDA for the Hubbard model. Since the spin density $n_{\sigma}(r)$ is the basic variable in the normal DF formalism, we use the spin occupation number $n_{i \sigma}$ of the orbital $i$ as the basic variable here [17]. We then write the XC energy functional as

$$
E_{\mathrm{xc}}\left\{n_{i \sigma}\right\}=C U \sum_{i \sigma} n_{i \sigma}^{4 / 3}
$$

neglecting the small modifications of the exchange only exponent $4 / 3$ by correlation effects. We choose $C=1 / 2$, which makes (3) correct for a spin-polarized $\mathrm{H}$ atom, since the LDA gives a rather accurate $\mathrm{XC}$ energy for a $\mathrm{H}$ atom.

The two-site Hubbard model can be solved exactly, by considering all configurations. The excitation energy to the optically allowed state is

$$
\Delta E_{\mathrm{ex}}=\frac{U}{2}\left[1+\sqrt{1+16\left(\frac{t}{U}\right)^{2}}\right]=U+4 \frac{t^{2}}{U}+t O\left(\frac{t^{3}}{U^{3}}\right) .
$$

The only one-particle excitation connects the bonding and antibonding orbitals. $\chi_{0}$ is then expressed in terms of products of these orbitals. Taking a matrix element of (1) between such a product, we obtain

$$
\begin{aligned}
\Delta E_{\mathrm{TDLDA}} & =\sqrt{\left(\omega_{0}\right)^{2}+4 \omega_{0}\left(\tilde{U}+\tilde{f}_{\mathrm{xc}}\right)} \\
& =\omega_{0}+2\left(\tilde{U}+\tilde{f}_{\mathrm{xc}}\right)+\omega_{0} O\left[\left(\frac{\tilde{U}+\tilde{f}_{\mathrm{xc}}}{\omega_{0}}\right)^{2}\right],
\end{aligned}
$$




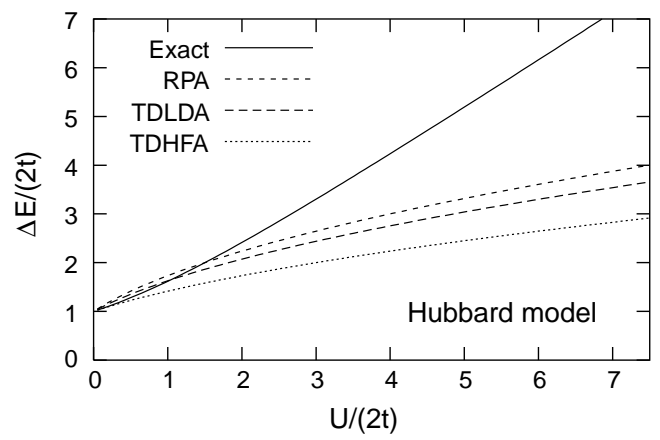

Fig. 2 - The excitation energy $\Delta E$ of the two-site Hubbard model as a function of $U /(2 t)$ in the exact calculation, the TDLDA, TDHFA and RPA.

where $\omega_{0}$ is the eigenvalue difference for the two orbitals and $\tilde{U}$ and $\tilde{f}_{\mathrm{xc}}$ are matrix elements between the orbital product of the Coulomb interaction and $f_{\mathrm{xc}}$, respectively. Here $\omega_{0}=2 t$, $\tilde{U}=U / 2$ and $\tilde{f}_{\mathrm{xc}}=-0.176 U$. Table I summarizes the results in the limit $U / t \ll 1$. In this limit the exact $\tilde{f}_{\mathrm{xc}}^{\mathrm{ex}} \approx-U / 4$, with only a weak energy dependence. We have also studied the time-dependent Hartree approximation or equivalently the random phase approximation (RPA), where $f_{\mathrm{xc}} \equiv 0$, as well as the time-dependent Hartree-Fock approximation (TDHFA). In this limit the RPA gives a much too large result, due to the unphysical self-interaction, while the self-interaction-free TDHFA gives the correct result. In TDLDA only a small part of the self-interaction is cancelled, and the RPA is not much improved.

The cancellation of the self-energy is fairly good for the XC energy (here by construction exact for a $\mathrm{H}$ atom), but poor for the TDLDA in the problem considered. The reason is the different density dependences in the approximate and exact exchange energies. In the LDA the density enters with the (approximate) power $4 / 3$, while in the exact XC energy it tends to have the power two. For instance, for unpolarized states of the two-site Hubbard model, the exact exchange energy is $-U \sum_{i \sigma} n_{i \sigma}^{2} / 2$, since in this case it just cancels the self-interaction. Taking the first density derivative to obtain the XC potential then gives a prefactor $4 / 3$ in the LDA but a prefactor 2 in the exact treatment. If the LDA XC energy was accurate, this then means that the $\mathrm{XC}$ potential is not. This was illustrated for the $\mathrm{H}$ atom [15]. To obtain $f_{\mathrm{xc}}$ we have to take another density derivative. This gives a prefactor $1 / 3$ in the TDLDA but unity in the exact treatment. The error in $f_{\mathrm{xc}}$ is then even larger, as illustrated in table I. The limit $U / t \ll 1$ corresponds to small values of $d$ for the $\mathrm{H}_{2}$ model. Then the Hubbard model is not a good model of $\mathrm{H}_{2}$, due to the importance of other Coulomb integrals than $U$.

Figure 2 shows the results of the Hubbard model. The results for $U /(2 t)<1$ were discussed in table I. For larger values of $U / t$, the exact excitation energy is substantially larger than

TABLE I - The excitation energy of the two-site Hubbard model according to various approximations for $U / t \ll 1$.

\begin{tabular}{lr}
\hline Method & Excitation energy \\
\hline LDA & $2 t$ \\
RPA & $2 t+U$ \\
TDLDA & $2 t+0.82 U$ \\
TDHFA & $2 t+0.5 U$ \\
Exact & $2 t+0.5 U$ \\
\hline
\end{tabular}


the result of the RPA or TDLDA. This is not due to the missing cancellation of the selfinteraction, as illustrated by the poor TDHFA result. In this limit the exact excitation energy is of the order $U$ while the RPA and TDLDA results are $\sim \sqrt{U t}$. This can be traced back to the structure of eq. (1), the fact that $\chi_{0}(\omega) \sim 1 /\left[\omega^{2}-\omega_{0}^{2}\right]$ and that $\omega_{0} \rightarrow 0$. This explains the failure of the TDLDA for large separations. We obtain

$$
\tilde{f}_{\mathrm{xc}}^{\mathrm{ex}}(\omega)=-\frac{1}{2}(U+t)+\frac{\Delta E_{\mathrm{ex}}}{8 \alpha^{2}}+\frac{\omega^{2}}{8}\left(\frac{1}{t}-\frac{1}{\alpha^{2} \Delta E_{\mathrm{ex}}}\right),
$$

where $\alpha^{2}=\left(1-U / \sqrt{U^{2}+16 t^{2}}\right) / 2$. For $U / t \gg 1, \tilde{f}_{\mathrm{xc}}^{\mathrm{ex}}(\omega)$ has a very strong energy dependence, i.e., $\tilde{f}_{\mathrm{xc}}^{\mathrm{ex}}(\omega=0) \sim U^{3} / t^{2}$ and $\tilde{f}_{\mathrm{xc}}^{\mathrm{ex}}\left(\omega=\Delta E_{\mathrm{ex}}\right) \sim U^{2} / t$. While the dependence on $U$ is linear for $U \ll t$, as expected, it becomes very strong for $U \gg t$. Similar results were obtained by Gritsenko et al. [12], who derived their results from a Heitler-London Ansatz. In the LDA we find $\tilde{f}_{\mathrm{xc}} \sim-U$. In this limit, the TDLDA then gives a $\tilde{f}_{\mathrm{xc}}$ which has not just an incorrect dependence on $U$ but even the wrong sign.

For the spin-polarized case and large $d$, the excitation energy is the eigenvalue difference $\omega_{0}$, which in this limit is the difference between the spin-up and spin-down eigenvalues for a spin-polarized atom. Since this difference is finite, it is a qualitative improvement. The excitation energy is, however, still substantially too small.

For $d \rightarrow 0, \mathrm{H}_{2}$ reduces to He atom and the bonding and antibonding orbitals approach $1 s$ and $2 p$ orbitals, respectively. The TDLDA gives essentially no correction to the eigenvalue difference for the He $1 s \rightarrow 2 p$ transition, due to the small overlap between the $1 s$ and $2 p$ wave functions. This is consistent with the results in fig. 1, where the TDLDA correction is further reduced by the fact that the antibonding LDA solution is unbound for $d \sim 1 a_{0}$.

For a somewhat larger $d$, the extent of the bonding and antibonding orbitals becomes comparable. It is then more natural to compare with Be, where $2 s$ and $2 p$ orbitals have a comparable extent. We assume that radial parts are identical, which is a very good approximation [18]. Considering the exchange only approximation and neglecting the core electrons, the integrals of $v$ and $f_{x}$ can be easily calculated. Using Slater orbitals [19], we find that the integrals are $0.161 \lambda$ and $0.069 \lambda$, respectively, where $\lambda$ is the exponent in the Slater orbital. Using the LDA value for the difference of the $2 p$ and $2 s$ orbital energies, $\omega_{0}=0.257$ Ryd, we obtain the TDLDA result $0.398 \mathrm{Ryd}$ for the $2 s$ to $2 p$ excitation. The full TDLDA calculation gives $0.363 \mathrm{Ryd}$ [8], the difference being due to the neglect of other excitations in the treatment above and the simplicity of the model. Using the same simplified formalism, we find that the excitation energy in the TDHFA is $0.386 \mathrm{Ryd}$, close to the experimental result 0.388 Ryd. These considerations illustrate that the model is fairly accurate, and we now use it to analyze why the TDLDA itself is rather accurate.

We consider a Be atom and calculate $\tilde{f}_{x}$ :

$$
\begin{aligned}
\tilde{f}_{x}^{\mathrm{Be}} & =\frac{\partial^{2} E_{x}^{\mathrm{np}}(n)}{\partial n^{2}}=\frac{1}{2} \frac{\partial^{2} E_{x}^{\mathrm{p}}(n)}{\partial n^{2}} \approx \\
& \approx \frac{1}{2}\left[E_{x}^{\mathrm{p}}(3)+E_{x}^{\mathrm{p}}(1)-2 E_{x}^{\mathrm{p}}(2)\right],
\end{aligned}
$$

where $E_{x}^{\mathrm{np}}(n)$ and $E_{x}^{\mathrm{p}}(n)$ are the exchange energies of $n$ electrons which are non-spin-polarized and spin-polarized, respectively. The first equality of (6) follows, since Be is non-spinpolarized, and the second equality follows from direct calculation. Ground-state energies of the type (6), where the shells are systematically filled up, are fairly well reproduced by the LDA [18], and we expect $\tilde{f}_{x}^{\mathrm{Be}}$ to be fairly accurate. Since correlation effects are small for 
the spin-polarized system, we expect the left-hand side of (6) to be similar to the HF result, which is

$$
E_{x}^{\mathrm{HF}}(3)+E_{x}^{\mathrm{HF}}(1)-2 E_{x}^{\mathrm{HF}}=-\frac{1}{3} G^{1}(2 s, 2 p)
$$

Here $G^{1}(2 s, 2 p)=\langle 2 s, 2 p|1 / r| 2 s 2 p\rangle$ is a Slater integral. Thus $\tilde{f}_{x} \approx-G^{1}(2 s, 2 p) / 6$. We furthermore have that $\tilde{U}=G^{1}(2 s, 2 p) / 3$. Assuming that the LDA eigenvalue difference $\omega_{0}=$ $\varepsilon_{2 p}-\varepsilon_{2 s}$ is the same as the Hartree difference, we obtain the exact excitation energy

$$
\Delta E=\omega_{0}+\frac{1}{3} G^{1}(2 s, 2 p)
$$

If (6) is fulfilled and if $\tilde{U}+\tilde{f}_{x} \ll \omega_{0}$, the TDLDA expression (4) is reduced to exactly (8). Thus we can understand why TDLDA is rather accurate for Be.

It is interesting to also analyze $\mathrm{Li}$. Since $\mathrm{Li}$ has just one valence electron and is spinpolarized, $\tilde{f}_{x}$ obtains an extra factor of two compared with the Be case. If the last part of eq. (6) is satisfied, we find that $\tilde{U}+\tilde{f}_{x}^{\mathrm{Li}}=0$, and the excitation energy is $\omega_{0}$. Since the effect of the core is small, $\omega_{0}$ should be close to the excitation energy. Thus if TDLDA is rather accurate for $\mathrm{Be}$, the same should also be true for $\mathrm{Li}$, as is indeed observed $[4,7,8]$.

We observe the difference between the Hubbard model and the Be atom. In the Hubbard model, the different density dependencies of the exact and LDA model exchange energies lead to the large error in the limit $U \ll t$. One might have expected similar problems in relating eqs. (6), (7), since we would expect the exact exchange energy to be $\sim n^{2} \bar{I}$, where $n$ is the number of electrons and $\bar{I}$ is an average exchange integral. However, $\bar{I}$ decreases with $n$ in such a way that the $n$ dependence is approximately $n^{4 / 3}[18]$. This is due to the increase in the number of nodes as states with higher values of the orbital quantum number $l$ are occupied. This is crucial for the fairly accurate TDLDA results for Li and Be. The same happens for $\mathrm{H}_{2}$ at intermediate $d$, since the antibonding orbital has an extra node. The effect is absent in the Hubbard model, due to the neglect of various Coulomb integrals which are important at small $d$. For larger values of $d$, however, these integrals are less important and the Hubbard model much more relevant.

It is very interesting to study the $2 s \rightarrow 2 p$ transition in the $\mathrm{F}$ atom, since the LSDA gives an exceptionally large error (about $0.20 \mathrm{Ryd}$ [18]) in the energy for this transition. This is due to the large difference in exchange interaction between a $2 s$ and a $2 p$ electron on the one hand, and the interaction between two $2 p$ electrons on the other hand, which cannot be described. In contrast, this transition is rather accurately described in the first half of first row. In the TDLDA we find the energy 1.47 Ryd for the $2 s \rightarrow 2 p$ transition of $\mathrm{F}$ compared with the experimental result $1.53 \mathrm{Ryd}$. The error, $0.06 \mathrm{Ryd}$, is relatively small, much smaller than in the LSDA $\Delta$ SCF calculation (0.20 Ryd) or from the eigenvalue difference (0.19 Ryd).

We have shown that the TDLDA gives a rather accurate description of the ${ }^{1} \Sigma_{g}^{+} \rightarrow{ }^{1} \Sigma_{u}^{+}$ excitation for intermediate nuclear separations $d$. For large $d$ 's the TDLDA fails qualitatively, while TDLSDA gives a finite excitation energy. This behaviour was compared with the Hubbard model, for which we found that $\tilde{f}_{\mathrm{xc}}^{\text {ex }}$ has a strong energy dependence for $U / t \gg 1$. For small and intermediate $d$ 's we compared with small atoms. The relatively accurate description of the $2 s \rightarrow 2 p$ excitation of $\mathrm{Li}$ and Be can be understood by relating it to ground-state energy differences. Our results for $\mathrm{H}_{2}$ points towards the need of a strongly energy-dependent $f_{\mathrm{xc}}$ to be able to treat the excitation energies in both the bonding and dissociation regimes. 
FA acknowledges support from the New Energy and Industrial Technology Development Organization (NEDO), OG the Max-Planck Forschungspreis and AR the Spanish MEC sabbatical program, the EC-Nanophase project and JCyL (VA28/99).

\section{REFERENCES}

[1] Hohenberg P. and Kohn W., Phys. Rev., 136 (1964) B864; Kohn W. and Sham L. J., Phys. Rev., 140 (1965) A1133.

[2] Runge E. and Gross E. K. U., Phys. Rev. Lett., 52 (1984) 997; Gross E. K. U. and Kohn W., Phys. Rev. Lett., 55 (1986) 2850; Ullrich C. A., Gossmann U. J. and Gross E. K. U., Phys. Rev. Lett., 74 (1995) 872.

[3] Zangwill A. and Soven P., Phys. Rev. A, 21 (1980) 1561; Stott M. J. and Zaremba E., Phys. Rev. A, 21 (1980) 12.

[4] Petersilka M., Gossmann U. J. and Gross E. K. U., Phys. Rev. Lett., 76 (1996) 1212.

[5] Casida M. E., in Recent Advances in Density-Functional Methods, edited by Chong D. P. (World Scientific, Singapore) 1995; Tokatly I. V. and Pankratov O., Phys. Rev. Lett., 86 (2001) 2078.

[6] See, e.g., van Gisbergen S. J. A., Snijders J. G. and Baerends E. J., Phys. Rev. Lett., 78 (1997) 3097; Casida M. E. et al., J. Chem. Phys., 108 (1998) 4439; van Gisbergen S. J. A., Groeneveld J. A., Rosa A., Snijders J. G. and Baerends E. J., J. Phys. Chem. A, 103 (1999) 6835; Ricciardi G., Rosa A., van Gisbergen S. J. A. and Baerends E. J., J. Phys. Chem. A, 104 (2000) 635; Cai Z. L., Tozer D. J. and Remer J. R., J. Chem. Phys., 113 (2000) 7084; Gonze X., Fuchs M., Klüner T. and Scheffler M., unpublished.

[7] March N. H., Rubio A. and Alonso J., J. Phys. B, 32 (1999) 2173.

[8] Vasiliev I., ÖGüt S. and Chelikowsky J. R., Phys. Rev. Lett., 82 (1999) 1919.

[9] Gadzuk J. W., Surf. Sci., 43 (1974) 44.

[10] Kolos W. and Wolniewicz L., J. Chem. Phys., 43 (1965) 2429; 45 (1966) 509.

[11] Casida M. E. et al., J. Phys. Chem., 113 (2000) 7062.

[12] Gritsenko O. V. et al., J. Chem. Phys., 113 (2000) 8478.

[13] Marques M. A. L., Castro A. and Rubio A., cond-mat/0102234 and to be published in $J$. Chem. Phys.

[14] Gunnarsson O. and Johansson P., Int. J. Quantum Chem., 10 (1976) 307.

[15] Gunnarsson O., Lundqvist B. I. and Wilkins J. W., Phys. Rev. B, 10 (1974) 1319.

[16] Gunnarsson O. and Lundqvist B. I., Phys. Rev. B, 13 (1976) 4274.

[17] Gunnarsson O. and Schönhammer K., Phys. Rev. Lett., 56 (1986) 1968.

[18] Gunnarsson O. and Jones R. O., Phys. Rev. B, 31 (1985) 7588; Jones R. O. and GunnarsSON O., Phys. Rev. Lett., 55 (1985) 107.

[19] Slater J. C., Phys. Rev. B, 36 (1930) 57. 